\title{
Rendimiento académico y factores educativos de estudiantes del programa de educación en entorno virtual. Influencia de variables docentes
}

\author{
Magaly M. Quiñones-Negrete ${ }^{1,2^{*}}$, Ana M. Martin-Cuadrado ${ }^{1}$ y Carmen R. Coloma-Manrique ${ }^{3}$ \\ (1) Universidad Nacional de Educación a Distancia (UNED), Escuela Internacional de Doctorado (EIDUNED), Programa \\ de Doctorado en Educación. C/Juan del Rosal, 14. Ciudad Universitaria 28040 Madrid- España. \\ (Correo-e: mquinones24@alumno.uned.es; amartin@edu.uned.es) \\ (2) Universidad Católica Los Ángeles de Chimbote, Jr. Tumbes 247, Chimbote-Perú. \\ (Correo-e: mquinonesn@uladech.edu.pe) \\ (3) Universidad Pontificia Católica del Perú. Av. Universitaria 1801- San Miguel 15088, Lima-Perú. \\ (Correo-e: crcoloma@pucp.edu.pe) \\ * Autor a quien debe ser dirigida la correspondencia.
}

Recibido Oct. 9, 2020; Aceptado Dic. 9, 2020; Versión final Feb. 8, 2021, Publicado Jun. 2021

\begin{abstract}
Resumen
El objetivo de esta investigación es explorar la relación de factores educativos (estrategias y estilos de aprendizaje) y competencias discentes genéricas (trabajo autónomo, cooperativo y competencia digital), mediados por dos variables docentes (competencia digital y estilo de enseñanza), en el rendimiento académico de los estudiantes en el entorno virtual de una universidad privada de Perú. Este es un estudio analítico-transversal, con una muestra de 216 estudiantes y 9 docentes de II a X ciclo del programa de educación. Se encontró que el estilo de aprendizaje, la competencia digital y el trabajo cooperativo fueron las variables con mayor peso. Los estilos de aprendizaje preferentes fueron el reflexivo y el activo; y, la estrategia más utilizada fue la búsqueda de selección. Como conclusión, se recomienda que los docentes continúen utilizando la plataforma digital y fortalezcan sus estilos de enseñanza, teniendo en cuenta el estilo de aprendizaje de los estudiantes, para facilitar un mayor rendimiento académico.
\end{abstract}

\section{Academic performance and educational factors of university students enrolled in a virtual environment education program. Influence of teaching variables}

\begin{abstract}
The objective of this research study is to explore the relationships between educational factors (learning strategies and styles) and generic student competences (autonomy, cooperative work, and digital competence), mediated by two teaching variables (digital competence and teaching style), in the academic performance of students enrolled in a virtual environment education program offered at a private university in Peru. This is a cross-sectional analytical study with a sample of 216 students and nine professors from the second to the tenth cycle of the education program. The results show that learning style, digital competence, and cooperative work are the most important variables. The most preferred learning styles are reflective and active and the most used strategy is selection searching. In conclusion, it is recommended that professors continue using digital platforms and that they strengthen their teaching styles, by taking into account student learning styles, to facilitate student academic performance.
\end{abstract}




\section{INTRODUCCIÓN}

El rendimiento académico es uno de los indicativos sobre el aprendizaje de los estudiantes en el proceso de enseñanza aprendizaje, siendo directamente relacionado con las calificaciones obtenidas para aprobar los exámenes establecidos de cada asignatura. La clasificación de las notas para evaluar el rendimiento académico, en el caso de Perú, se puede tomar como bien logrado (15 a 20 puntos); en otros casos, se denominaría regularmente logrado (11 a 14 puntos) o deficiente ( 0 a 10 puntos) (Colonio, 2017). Hay variedad de factores educativos que se relacionan con la mejora del rendimiento académico, como las estrategias y los estilos de aprendizaje (Norzagaray et al., 2013) (Dağ y Gecer, 2009); del mismo modo, las competencias genéricas: competencia digital, trabajo autónomo y cooperativo (Chou y Liu, 2005) (GarcíaValcárcel et al., 2009), quienes también forman parte del perfil del egresado en educación superior, que le capacitan como profesional y ciudadano, de tal modo que se deberá desarrollar a lo largo de sus estudios.

Las estrategias de aprendizaje se perciben como parte de los recursos que los estudiantes deben conocer y en las que deben participar para ser competentes. En pocas palabras, los estudiantes calificados 0 talentosos fracasan en la universidad o abandonan los estudios cuando no usan adecuadamente las estrategias de aprendizaje (Khan y Rasheed, 2019). Conocer las estrategias de aprendizaje que aplican los estudiantes durante su proceso de aprendizaje proporciona información sobre su forma de pensar y resolver problemas. Sirve de pistas para el docente, es decir son facilitadoras del acto didáctico, Los estilos de aprendizaje son las formas individuales que utilizan los estudiantes para procesar información y nuevos conceptos, y para desarrollar sus habilidades y destrezas durante el proceso de aprendizaje (Arbuthnott, et al., 2015). Conocer los tipos de estilos de aprendizaje que utilizan los estudiantes, permite a los docentes orientar el uso adecuado de los mismos ante la variedad de actividades y tares que supone el aprender. El docente adaptará su método de enseñanza de acuerdo al estilo de aprendizaje del estudiante, de tal modo que contribuya a la mejora del rendimiento académico (Rahimi, et al., 2017).

Las competencias genéricas sirven a los estudiantes universitarios para convivir con un mundo cada vez más global, versátil y digitalizado; el predominio de la tecnología y el cambio continuo de la sociedad exigen competencias para aprender a aprender y para convivir, especialmente; estas habilidades son multifuncionales y, una vez adquiridas, se aplican en diferentes disciplinas de aprendizaje y situaciones de trabajo; son muchas las competencias genéricas, pero, para este estudio se consideraron: trabajo cooperativo, trabajo autónomo y competencia digital ( $\mathrm{Ng}$ y So, 2017). La competencia de trabajo cooperativo o trabajo en equipo supone la interacción y colaboración del estudiante con sus compañeros de grupo para lograr una tarea común, Tang, (2020), encontraron que las competencias de trabajo en equipo o trabajo cooperativo en las aulas se relacionan con la mejora del rendimiento académico. Para Mari y Gumel., (2015), la estrategia de aprendizaje cooperativo es un método de enseñanza que se debe usar en las asignaturas de las facultades de educación porque tiene la capacidad de mejorar el rendimiento académico, y satisfacer las necesidades de aprendizaje de sus estudiantes.

El trabajo autónomo se refiere al desarrollo de la capacidad de reflexionar sobre los propios procesos de aprendizaje y orientarlos hacia el logro de objetivos; es decir, el estudiante tiene que ser independiente y ser gestor activo de su propio aprendizaje. Para Gonzalez et al., (2020), los estudiantes que aprenden de manera autónoma desarrollan con precisión las actividades mediante el uso de herramientas tanto en el aprendizaje como en la evaluación. Asimismo, "la autonomía del estudiante se relaciona positivamente con experiencias de aprendizaje exitosas y satisfacción con la vida" (Henri, 2018).

Finalmente, para llomäki et al. (2016), la competencia digital se refiere a las habilidades y alfabetizaciones necesarias para que el ciudadano medio pueda aprender y navegar en la sociedad del conocimiento digitalizada. Por lo demás, las tecnologías digitales se están convirtiendo en la parte central del trabajo diario en el ejercicio profesional. Este desafío genera demandas considerables para las instituciones de educación superior en lo que respecta al desarrollo de estrategias para respaldar las competencias digitales necesarias para proporcionar una enseñanza y un aprendizaje de alta calidad (Pettersson, 2018). El desarrollo de la competencia digital no se adquiere sólo interactuando con los medios digitales; sino que, es primordial la generación de procesos educativos que fomenten la adquisición de la competencia digital por parte de los estudiantes (Pascual et al., 2019).

Se debe tener en cuenta que las habilidades digitales y los estilos de enseñanza del docente son variables que afectan la relación de los factores educativos del estudiante y su rendimiento académico (Zempoalteca et al., 2017). Por otro lado, Las Tecnologías de Información y Comunicación (TIC) están generando un impacto positivo en la educación superior, mediante la innovación del proceso de enseñanza aprendizaje y la accesibilidad a las herramientas y escenarios utilizados en los procesos de implementación de estrategias didácticas, con la finalidad de facilitar la construcción del conocimiento y desarrollar competencias (Martínez-Palmera, et al., 2018), donde las herramientas más usadas son los Entornos Virtuales de Aprendizaje (EVA). 
Un entorno virtual de aprendizaje (EVA) es una aplicación informática diseñada para facilitar la comunicación pedagógica entre estudiantes y docentes que participan en el proceso de enseñanza aprendizaje (Silva, 2017). Las instituciones de educación superior en países desarrollados y en desarrollo están logrando un progreso notable en la gestión de la plataforma Moodle como herramienta de aprendizaje para la difusión y adquisición de conocimientos. Sin embargo, esta práctica ha recibido más atención en los países desarrollados que en los países en desarrollo. Asimismo, la conectividad y el acceso a Internet deben estar disponibles tanto para los estudiantes como para los docentes de las instituciones de educación superior para que todos puedan hacer uso de la plataforma virtual. Al concluir la investigación, los resultados mostraron que los discentes tienen habilidades en el uso de herramientas digitales, y en el desarrollo del trabajo en cooperativo. Asimismo, cuando se evalúan de manera conjunta las variables: estilo de aprendizaje, trabajo cooperativo, y competencia digital se demuestra que influyen en el rendimiento académico dentro de un entorno virtual. Por otro lado, las variables moderadoras docentes dentro de un entorno virtual presentaron un nivel alto en estilo de enseñanza y competencia digital.

\section{METODOLOGÍA}

La metodología del presente estudio es de tipo analítico-transversal y se utilizaron cuestionarios validados para la evaluación de las variables discentes y docente; a excepción del rendimiento académico que proviene de la nota final de las asignaturas de los docentes participantes del estudio. Se evaluaron algunos factores educativos del estudiante que influyen en el rendimiento académico, mediados por variables docentes dentro de un entorno virtual de una universidad del Perú a través de regresión lineal múltiple y la información fue recolectada en estudiantes del programa de educación El presente estudio primario se fundamenta en el paradigma cuantitativo, en este marco se desarrolló un estudio analítico de corte transversal no experimental, la metodología empleada para la selección, recolección, procesamiento y análisis de información del estudio se declara de manera resumida en los siguientes 5 pasos: (1) Participantes, (2) Variables e Instrumentos de medición, (3) Recolección de información, (4) Modelos estadísticos y (5) Análisis estadístico.

\section{Participantes}

En este estudio se determinó como participantes a los docentes y estudiantes del Programa de Educación de una universidad privada de Perú con enseñanza a distancia, ubicada en la ciudad de Chimbote. La población estuvo conformada por 428 estudiantes del segundo semestre de estudios del año 2019 (especificando que, en el Perú, la mayoría de universidades dividen el año lectivo en dos cuatrimestres), provenientes del segundo hasta el décimo ciclo de estudios; además, 12 docentes a tiempo completo de las principales asignaturas del Programa de Educación. La muestra de estudiantes se calculó utilizando un muestreo probabilístico estratificado con fijación proporcional, con el $95 \%$ de nivel de confianza, $5 \%$ de error de muestreo y probabilidad del 50\%, resultando una muestra de 216 estudiantes, véase la distribución en la Tabla 1. Mientras que, para el cálculo de muestra de docentes se utilizó un muestreo no probabilístico por conveniencia conformado por 09 docentes (un docente por cada ciclo de estudio).

Tabla 1: Distribución del tamaño de población y muestra de estudiante, estratificado según ciclo de estudio.

\begin{tabular}{|c|c|c|}
\hline Ciclo de estudio & Población & Muestra \\
\hline 2 & 188 & 95 \\
\hline 3 & 38 & 19 \\
\hline 4 & 45 & 23 \\
\hline 5 & 45 & 23 \\
\hline 6 & 32 & 16 \\
\hline 7 & 20 & 10 \\
\hline 8 & 26 & 13 \\
\hline 9 & 18 & 9 \\
\hline 10 & 16 & 8 \\
\hline Total & 428 & 216 \\
\hline
\end{tabular}

Los criterios de inclusión de los participantes en el estudio fueron, respecto a los estudiantes, que debían estar correctamente matriculados y activos dentro del programa a distancia (virtual) en el semestre 2019-II; en cuanto a los docentes, debían trabajar a tiempo completo y utilizar el entorno virtual como medio de enseñanza. Tanto, los estudiantes como los docentes aceptaron voluntariamente participar del estudio.

\section{Variables e instrumentos}

El estudio utilizó tres tipos de variables: dependiente, independientes y moderadoras. La variable dependiente fue el rendimiento académico. Las variables independientes fueron las variables estrategia de 
aprendizaje, estilo de aprendizaje y competencias genéricas del discente (trabajo autónomo, trabajo cooperativo y competencia digital) y sus respectivas dimensiones. Las variables moderadoras fueron el estilo de enseñanza y competencia digital del docente. Para la recolección de las variables se utilizó 01 ficha de información y 07 instrumentos de medición validados y adaptados a población universitaria, los cuales se describen a continuación:

El rendimiento académico está definido como la nota obtenida por el estudiante en una determinada materia, curso o semestre. Para la presente investigación se obtuvo a partir de la nota promedio del estudiante al final del ciclo académico dentro de las principales asignaturas del Programa de Educación. El estilo de aprendizaje siendo definido como rasgos cognitivos, afectivos y fisiológicos de los estudiantes que sirven como indicadores relevantes de respuesta en los ambientes de aprendizaje; dividido según el modelo planteado por Honey y Mumford en activo, reflexivo, teórico y pragmático.

Se utilizó el cuestionario realizado por Honey-Alonso (CHAEA), diseñado por Alonso, Gallego y Honey en el año 1997 en España, con una escala de medición dicotómica $(0=\mathrm{No} ; 1=\mathrm{Si})$ y donde originalmente el instrumento consta de 80 ítems, distribuidos en 4 dimensiones acorde a los estilos de aprendizaje (activo, reflexivo, teórico y pragmático);sin embargo, un estudio realizado en México mostro validez y confiablidad de dicho reduciéndolo a 36 ítems utilizando análisis factorial exploratorio reportando los estadísticos KMO altos $(0.70)$ y prueba de esfericidad de Bartlett $(p<0.00)$ y varianza explicada $(48 \%)$; asimismo, se reportó una buena confiabilidad según dimensiones $\left(\alpha_{\min }=0.54 ; \alpha_{\max }=0.58\right)$ (Juárez, 2014).

La estrategia de aprendizaje se define como herramienta útil del estudiante para afrontar el proceso de enseñanza-aprendizaje. Se utilizó el cuestionario de evaluación de estrategias de aprendizaje (CEVEAPU), creado por Gargallo, Suarez y Pérez en el 2009 en España que originalmente consta de 167 ítems en una escala de medición de 5 categorías ( $1=$ Muy en desacuerdo; 5 = Muy de acuerdo). Asimismo, un estudio realizado en España mostró que el instrumento en la versión español tiene alta confiabilidad $(\alpha=0.90)$ y según dimensiones $\left(\alpha_{1}=0.82 ; \alpha_{2}=0.86\right)$ con 88 ítems; además presentó buena validez a través del análisis factorial exploratorio con rotación Varimax reportando los índices estadísticos KMO y esfericidad de Bartlett significativos $(p<0.00)$. (Gargallo et al., 2009).

Las competencias genéricas discentes se definen como las habilidades, conocimientos y acciones que desarrolla el estudiante para desarrollar sus tareas profesionales, donde el presente estudio se centró en tres competencias: el trabajo autónomo, trabajo cooperativo y la competencia digital. Para el primero se utilizó el cuestionario de Estrategia de trabajo autónomo (CETA) creado por López-Aguado en el año 2010 en España compuesto originalmente de 48 ítems en escala de Likert de 5 dimensiones ( $1=$ Nunca; 5 = Siempre) divido en 5 dimensiones (estrategias de ampliación, estrategia de colaboración, estrategia de conceptualización, estrategia de planificación y de preparación de exámenes); asimismo, reportó una alta confiabilidad del instrumento global $(\alpha=0.89)$ pero perdiendo un poco el poder estadístico al ser medido por dimensiones $\left(\alpha_{\min }=0.66 ; \alpha_{\max }=0.85\right)$; asimismo, mostró buena validez a través del Análisis Factorial Exploratorio reportando valores significativos en el estadístico $\mathrm{KMO}$ y cargas factoriales mayores de 0.4 (López-Aguado, 2010).

El segundo utilizó el cuestionario de Evaluación de Análisis del trabajo cooperativo en educación superior (ACOES), creado por García, Gónzalez y Mérida en el 2012 en España con una versión original de 52 ítems en 7 dimensiones teniendo como escala de Likert de 5 dimensiones ( $1=$ Total desacuerdo; $5=$ Totalmente de acuerdo), pero un estudio realizado en España de validez y confiabilidad mostró que a través del Análisis Factorial Confirmatorio solo eran consistentes 49 ítems y reportando una alta confiabilidad $(\alpha=0.89)$ del instrumento total y 2 según dimensiones ( $\alpha_{\min }=0.76 ; \alpha_{\max }=0.84$ ) a través del Alfa de Cronbach (García et al.,2012).

El tercero utilizó el cuestionario de Competencias básicas digitales 2.0 de los estudiantes universitarios (COBADI), creado por Zapata en el año 2013. El instrumento está conformado 56 ítems conformado en 6 dimensiones ( Medios tecnológicos, Uso de tiempo del internet, Competencia de conocimiento y uso de las TIC en la comunicación y trabajo colaborativo, Competencias de uso de las TIC para la búsqueda y tratamiento de la información, Competencias interpersonales en el uso de las TIC en el contexto universitario y Herramientas virtuales y de comunicación social en el Pregrado), este instrumento reporta validez de contenido por juicio de expertos (Veytia, 2013).

Mientras que, las variables moderadoras o covariables del estudio son las referidas por el docente: la competencia digital y el estilo de enseñanza, donde la primera muestra las habilidades, conocimientos y actitudes digitales para programar, desarrollar y evaluar sus asignaturas dentro de los entornos virtuales; mientras que, el segundo diagnostica las estrategias docentes en cuanto al acto didáctico. La competencia digital docente se midió utilizando el cuestionario de Autopercepción de la Competencia Digital Docente (CACDD), creado por Esteve-Mon en 2015 en España basado en los estándares de la sociedad 
internacional para la tecnología en educación (ISTE) y consta de 40 preguntas en escala de Likert de 8 categorías validado a través de validez de contenido por juicio de expertos (Esteve-Mon, 2015).

Por otro lado, respecto a la variable docente, estilo de enseñanza, se midió utilizando el cuestionario de Evaluación de la metodología docente y evaluativa de los profesores (CEMEDEPU)de 51 ítems en escala de medición de 5 categorías, creado por Gargallo, Rodríguez, Garfella y Fernández en el 2011 en España y fue validado a través del análisis factorial confirmatorio presentando aceptables indicadores de bondad de ajuste y alta confiabilidad según dimensiones ( $\left.\alpha_{\min }=0.84 ; \alpha_{\max }=0.86\right)$ (Gargallo et al., 2011).

La escala de medición de las variables de estudio fue numérica para los análisis de los modelos estadísticos, dado que, para el rendimiento académico se utilizó el promedio de notas finales; mientras que, en las variables independientes y moderadoras se utilizaron los puntajes obtenidos por dimensiones e instrumento total, los cuales se describen en la siguiente etapa. Todos los instrumentos internacionales fueron validados para el presente estudio a través de constructo interno utilizando análisis factorial confirmatorio reportando aceptables valores de índices de bondad de ajuste (CFI,TLI $\geq 0.90$; RMSEA $\leq 0.08$ ); mientras que, la fiabilidad de los instrumentos se obtuvo a través del alfa de Cronbach ( $\alpha$ ) reportando regularmente buenos valores estadísticos $(\alpha \geq 0.50)$ (Anexo Suplementario 1).

\section{Recolección de información}

La recolección de datos se realizó en una sesión presencial entre los meses de agosto y octubre del año 2019, a estudiantes del segundo a décimo ciclo, previamente, se solicitó el permiso de las autoridades y docentes del Programa de Educación. Teniendo la aprobación respectiva, se les explicó a los participantes el objetivo de estudio, su participación anónima y la confidencialidad de la información. Posteriormente, los estudiantes y docentes que aceptaron participar del estudio, tuvieron que firmar el consentimiento informado. La realización de la evaluación fue individual y tuvo una duración aproximada de 60 minutos.

\section{Modelos estadísticos}

Se generaron dos modelos estadísticos utilizando regresión lineal múltiple para evaluar la influencia de las variables estudiantiles (variables macro) y sus dimensiones (sub variables) con la variable dependiente, teniendo en cuenta la influencia de las variables moderadoras dentro de un entorno virtual. Para el análisis se tuvo en cuenta el p-valor, el cual explica la probabilidad de aceptación de la hipótesis nula, en comparación con el nivel de significancia $(\alpha=0.001,0.05)$. En seguida se muestra el trabajo especificado según los modelos bajo la función que se indica en la ecuación 1 y 2, también graficada a nivel general en la figura 1:

Rendimiento académico $=f$ (estrategias de aprendizaje, estilo de aprendizaje, trabajo autónomo, trabajo cooperativo, competencia digital estudiantil, estilo de enseñanza docente, competencia digital docente)

Rendimiento académico $=f$ (dimensiones de las variables estudiantiles, competencia digital docente, estilo de enseñanza docente)

Los modelos teóricos generados en la ecuación 3 y 4 para analizar el rendimiento académico, teniendo en cuenta las variables estudiantiles $\left(\beta_{i}\right)$ y sus dimensiones $\left(\beta_{x}\right)$ (variables independientes), teniendo la influencia del estilo de enseñanza y competencia digital docente (variables moderadoras) dentro de un entorno virtual.

Rendimiento académico $=\beta_{\mathrm{i}}$ Variables estudiantil $+\beta_{2}$ Estilo de enseñanza $+\beta_{3}$ Competencia digital docente $+\mu_{i}$

Rendimiento académico $=\beta x$ Dimensiones de variables estudiantil $+\beta_{2}$ Estilo de enseñanza $+\beta_{3}$ Competencia digital docente $+\mu_{\mathrm{i}}$

\section{Análisis Estadístico}

El procesamiento de datos se realizó en el programa Microsoft Excel 2016, en el cual se generó una base de datos y se recategorizó las variables de estudio para reportarlos según niveles de la siguiente manera: 1) variable dependiente (rendimiento académico), según la Dirección de Educación Básica Regular (deficiente (0-10), regularmente logrado (11-14) y bien logrado (15-20) (Digebare, 1980; citado por Colonio, 2017) y 2) variables independientes y moderadoras, a través de baremación por percentiles. Posteriormente, se importó para el análisis de datos al programa estadístico Stata 15.0. Los análisis que se realizaron fueron dados en dos etapas: 1) análisis descriptivo, donde se reportó frecuencias/ porcentajes con la finalidad de caracterizar según niveles las variables de estudio; 2) análisis correlacional, para evaluar las variables estudiantiles y dimensiones que influyen en el rendimiento académico, teniendo como variables 
moderadoras al estilo de enseñanza y competencia digital docente dentro de un entorno virtual; para este análisis todas las variables tuvieron una escala de medición numérica y se utilizó el modelo de regresión lineal mutiple con varianza robusta reportando beta y con intervalo de confianza al 95\%(IC95\%); siendo significativas con $\mathrm{p}<0.05$.

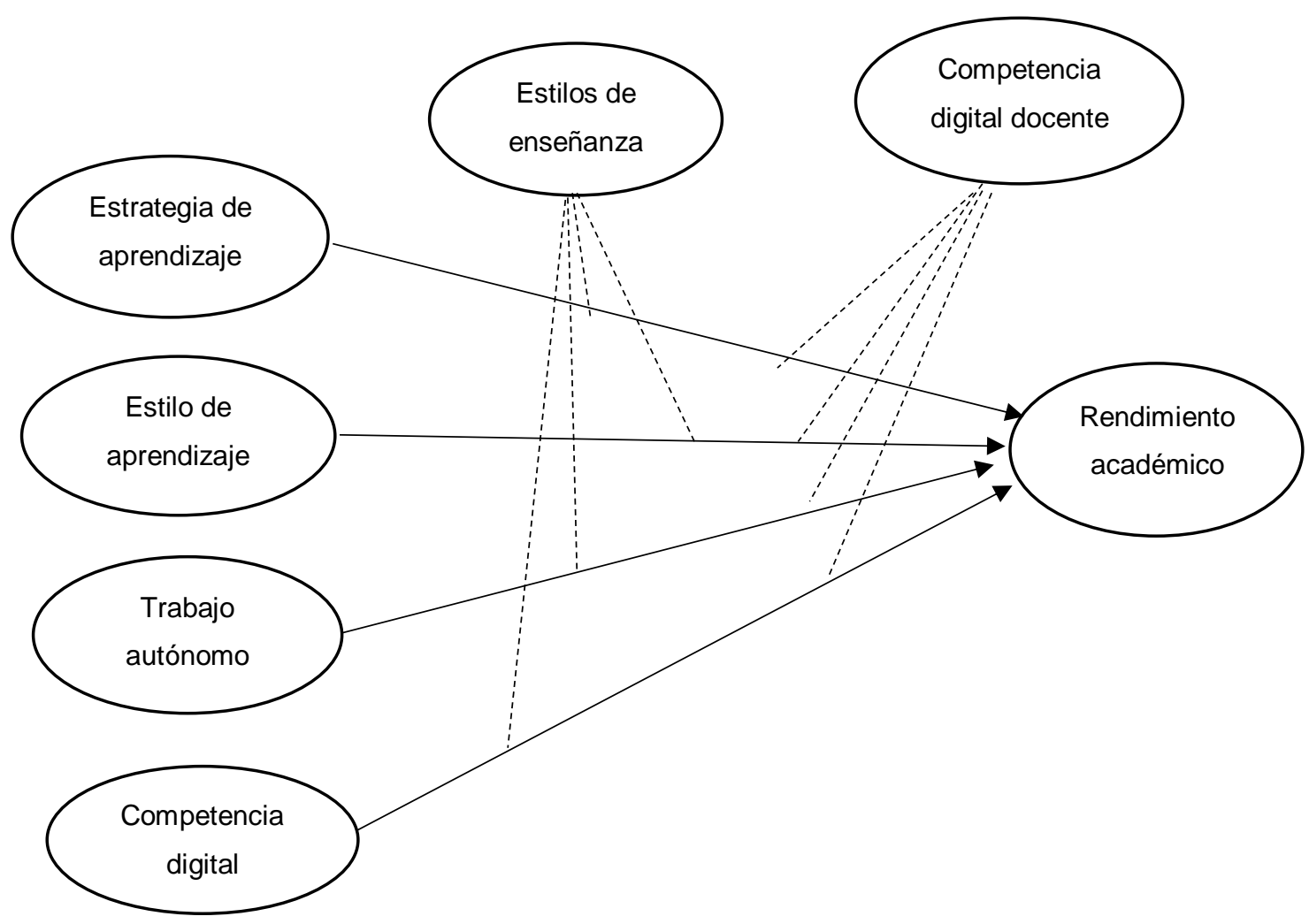

Fig.1: Variables utilizadas en el modelo

\section{RESULTADOS Y DISCUSIÓN}

En la Figura 2 se observó que los estudiantes de una universidad privada de Perú en el año 2019 dentro de un entorno virtual reportaron tener un bajo nivel en estrategias de aprendizaje (51.85\%). En la Figura 3 los estudiantes reportaron información del estilo de aprendizaje, nivel alto en el estilos de aprendizaje teórico (55.56\%) y nivel medio en el estilo de aprendizaje reflexivo (55.07\%) y pragmático $(50.00 \%)$; mientras que, el estilo de aprendizaje activo reportó un nivel bajo (51,39\%).En la Figura 4 se reportó que los estudiantes presentaron nivel alto en las competencias genéricas: trabajo autónomo (50.93\%), cooperativo (48.61\%), y competencia digital $(56.02 \%)$. Algunos de estos resultados obtenidos, coinciden con lo reportado por otro estudio como el realizado por Esteve-Mon et al. (2020); donde presentaron nivel alto en estudiantes universitarios en estilo teórico de aprendizaje; trabajo autónomo cooperativo y competencia digital; sirviendo estas habilidades para un mejor desenvolvimiento académico. Todo lo mencionado nos muestra que los estudiantes en la actualidad están inmersos dentro del ámbito virtual, es decir, desarrollan con mayor facilidad destrezas y habilidades para aprender a aprender dentro de dicho medio, y adaptarse a las necesidades del mercado laboral.

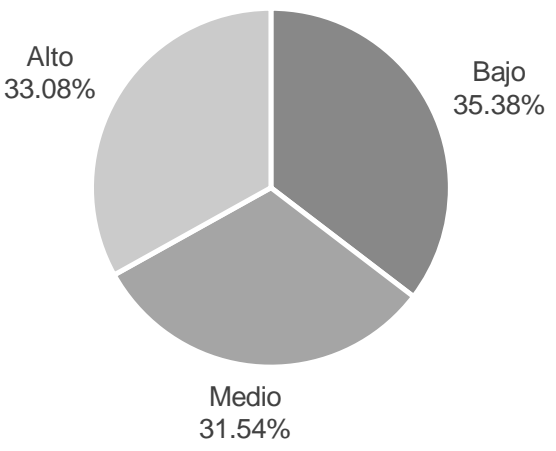

Fig. 2: Caracterización de estrategias de aprendizaje de los estudiantes. 


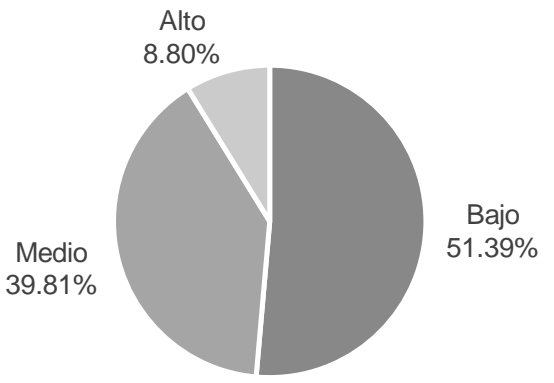

A)

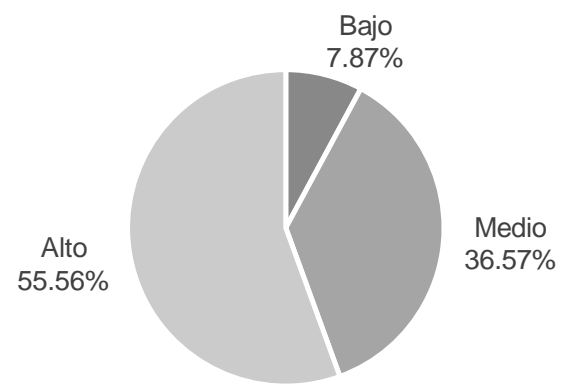

C)
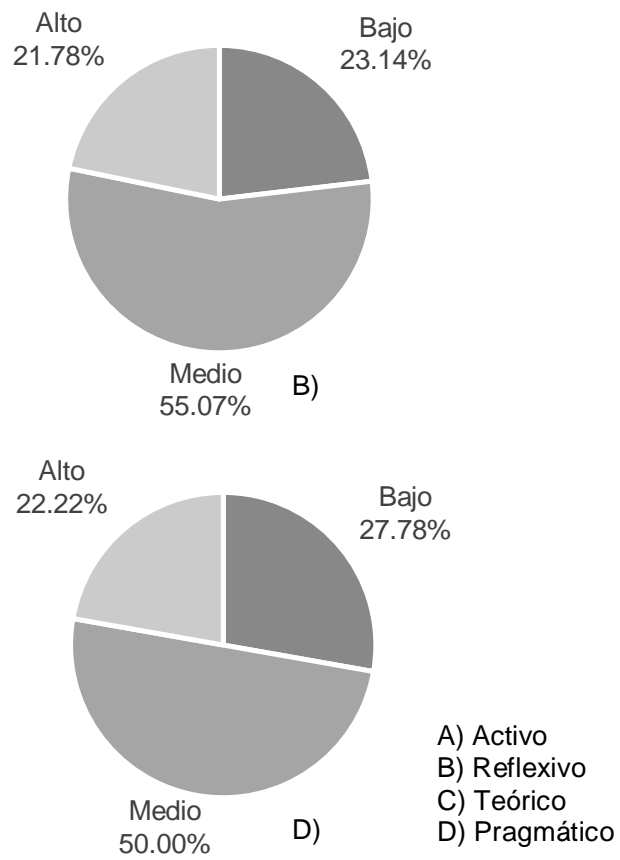

Fig. 3: Caracterización de estilos de aprendizaje de los estudiantes

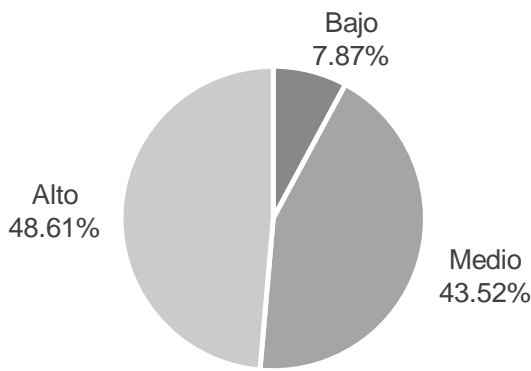

A)

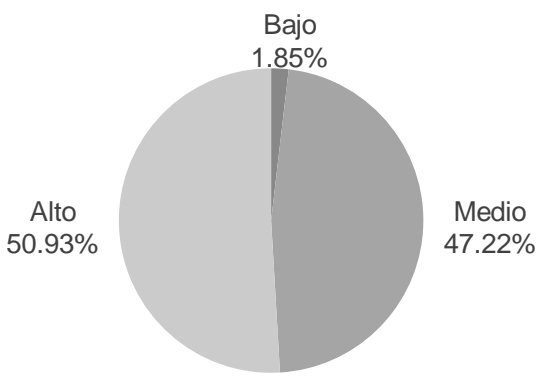

B)

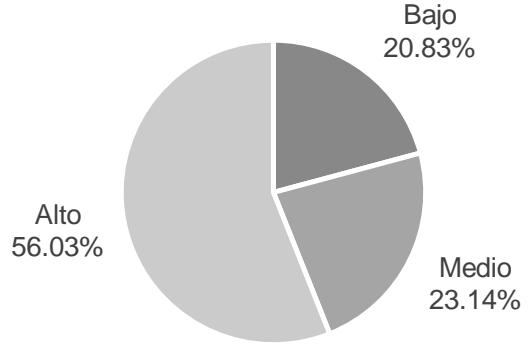

C)
A) Trabajo cooperativo

B) Trabajo autónomo

C) Competencia Digital

Fig. 4: Caracterización de las competencias genéricas de los discentes.

En la Figura 5 se reportó el rendimiento académico de los estudiantes dentro de un entorno virtual con un nivel alto, donde la mayoría se ubicó dentro de la máxima categoría (bien logrado) con $80.09 \%$. Estudio con resultado similar realizado en Estados Unidos reportó que el estudiante responde mejor dentro de un entorno virtual en comparación a un medio presencial (Hughes et al., 2007). Lo expuesto podría explicarse en que, la universidad de estudio y muchas de las universidades en el mundo han incorporado políticas orientadas a incorporar el currículo por competencias, inclusión de capacitaciones y uso en los entornos virtuales para el proceso de enseñanza-aprendizaje durante toda la formación profesional del estudiante, logrando superar los obstáculos naturales que se genera dentro de este medio de enseñanza y obteniendo una mejor respuesta académica. 


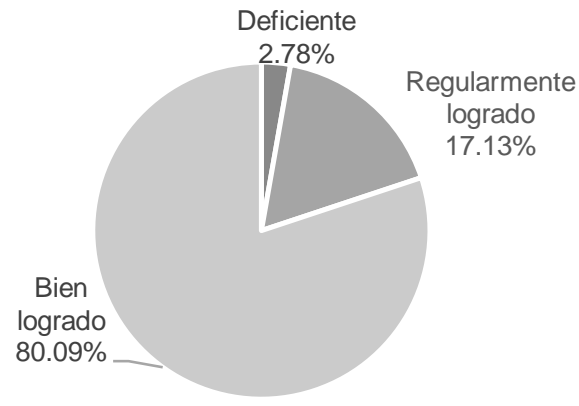

Fig. 5: Caracterización del rendimiento académico de los estudiantes.

Por otro lado, las variables moderadoras docentes dentro de un entorno virtual presentaron nivel alto en estilo de enseñanza a través de la evaluación metodológica docente y evaluativa (44.45\%) y competencia digital (44.45\%), identificando que los docentes de dicha institución tienen un perfil bueno y alto de enseñanza, así como un nivel alto en el uso de tecnologías para la enseñanza de sus asignaturas ( Figura 6 y 7), resultados que concuerdan con el estudio realizado por Gómez-Galán (2020), donde reportó que los docentes universitarios tienen un buen nivel en competencias digitales, siendo un factor condicionante para un mayor uso de las TIC en el proceso de enseñanza y mejora de la competencia digital del estudiante; asimismo, con respecto al estilos docentes en lo pedagógico (preparación y desarrollo de sus clases), interacción educativa y profesionalismo. Todo ello, se debe a que los docentes están en una constante formación continua, y además que muchas de las universidades a nivel mundial están exigiendo como requisito que los docentes tengan una alta destreza con las Tecnologías de la Información y Comunicación (TIC).

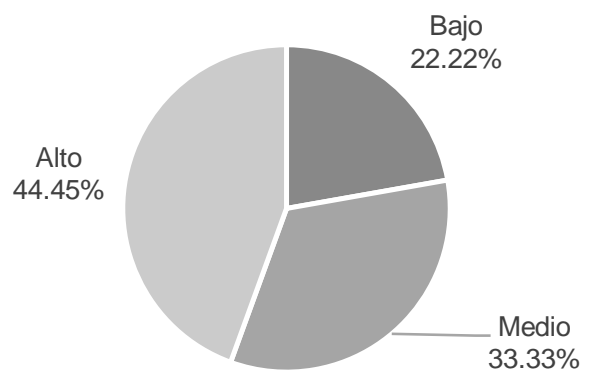

Fig. 6: Caracterización de la competencia digital docente

En la tabla 2 y 3 se reportaron las estimaciones en función de los modelos de regresión lineal múltiple, para evaluar las variables educativas del estudiante y sus dimensiones, influyentes en el rendimiento académico y teniendo como moderadoras las variables del docente dentro de un entorno virtual. Previamente, ambos modelos fueron evaluados si son adecuados y sus coeficientes son diferentes de cero, reportaron un $\mathrm{p}$ valor de la prueba $F$ resultante menor a 0.05 y rechazando la hipótesis nula; es decir los modelos son aceptados. Posteriormente, se presenta marca de asterisco para reportar la significancia del $p$-valor ( ${ }^{*}$ significativa menor al 5\%, ${ }^{* *}$ Significativa menor al $1 \%$ ), valor beta e intervalo de confianza al $95 \%$. Se encontró que según las variables educativas del estudiante del programa de Educación, a mayor habilidades de forma independiente el estilo de aprendizaje $(\beta=0.041 ; p<0.05)$, la competencia digital $(\beta=0.013 ; p<0.05)$ y el trabajo cooperativo $(\beta=0.010 ; p<0.05)$ de los estudiantes aumentan su rendimiento académico (Tabla 2$)$. Mientras que, nuestros resultados contrastan con otros estudios como el realizado por Colonio (2017) en el Perú, en el que se indica que el estilo de aprendizaje no influye en el rendimiento académico dentro un entorno presencial, de estos resultados contrastados se deriva que las universidades debe buscar tener una capacitación continuas para tipo de destrezas en el estudiante y docente teniendo mayor énfasis quienes tienen y realizan educación a distancia mejorando la motivación de estudios, calidad de enseñanzaaprendizaje en el estudiante.

Tabla 2: Relación de variables con el rendimiento académico en estudiantes (influencia de los estilos docentes y competencia digital)

\begin{tabular}{|l|c|c|}
\hline \multicolumn{1}{|c|}{ Variables } & $\beta$ & {$[I C 95 \%]$} \\
\hline Estilo de aprendizaje & $0.041^{*}$ & {$[0.063 ; 0.075]$} \\
\hline Trabajo Cooperativo & $0.010^{*}$ & {$[0.002 ; 0.018]$} \\
\hline Estrategia de aprendizaje & 0.002 & {$[-0.003 ; 0.008]$} \\
\hline Trabajo autónomo & 0.006 & {$[-0.001 ; 0.012]$} \\
\hline Competencia digital estudiante & $0.013^{* *}$ & {$[0.005 ; 0.020]$} \\
\hline $\mathrm{R}^{2}=11.4 \%, \mathrm{~F}: 2.93 \mathrm{p}<0.05$ & \multicolumn{3}{|c|}{} \\
\hline
\end{tabular}


Asimismo, de la Tabla 3 se reportó las dimensiones de las variables macro que tienen mayor aporte y aumentan el rendimiento académico. Dentro de la competencia digital, uso de herramientas virtual y comunicación social en pregrado $(\beta=0.222 ; p<0.001)$, conocimiento y uso de las TIC en la comunicación $(\beta=0.122 ; p<0.05)$ y trabajo en equipo uso de medios tecnológicos $(\beta=0.117 ; p<0.05)$ aumentan el rendimiento académico. Asimismo, el estilo de aprendizaje activo $(\beta=0.126 ; p<0.001)$ y reflexivo $(\beta=0.123$; $p<0.001)$ aumentan el rendimiento académico. El trabajo cooperativo en donde los estudiantes influye en la concepción del trabajo en grupo $(\beta=0.066 ; p<0.05)$ y su utilidad dentro trabajo en grupo para su formación $(\beta=0.057 ; p<0.05)$ aumentan el rendimiento académico. En las estrategias de aprendizaje, los estudiantes con mayor estrategia de búsqueda de proceso de información y selección $(\beta=0.060,0.049 ; p<0.05)$. 3) trabajo cooperativo, influye en la concepción del trabajo en grupo $(\beta=0.066 ; p<0.05)$ aumenta el rendimiento académico.

Tabla 3: Relación de las dimensiones de las variables independientes con el rendimiento académico en estudiantes teniendo la influencia del estilo docente y su competencia digital.

\begin{tabular}{|c|c|c|c|}
\hline Variables & Dimensión & $\beta$ & [IC95\%] \\
\hline \multirow{4}{*}{$\begin{array}{l}\text { Estilo de } \\
\text { aprendizaje }\end{array}$} & Activo & $0.126^{* *}$ & {$[0.044 ; 0.207]$} \\
\hline & Reflexivo & $0.123^{* *}$ & {$[0.041 ; 0.205]$} \\
\hline & Teórico & -0.078 & {$[-0.177 ; 0.021]$} \\
\hline & Pragmático & 0.028 & {$[-0.065 ; 0.122]$} \\
\hline \multirow{7}{*}{$\begin{array}{l}\text { Trabajo } \\
\text { Cooperativo }\end{array}$} & Concepción del Trabajo en grupo & $0.066^{*}$ & [0.020;0.112] \\
\hline & Utilidades del trabajo en grupo para su formación & $0.057^{*}$ & {$[0.010 ; 0.104]$} \\
\hline & Planificación del trabajo de los grupos por parte del profesorado & 0.018 & {$[-0.037 ; 0.072]$} \\
\hline & Criterios para organizar los grupos & $0.054^{* *}$ & {$[0.025 ; 0.084]$} \\
\hline & Normas de los grupos & 0.030 & {$[-0.002 ; 0.062]$} \\
\hline & Funcionamiento interno & $0.049^{*}$ & {$[0.011 ; 0.088]$} \\
\hline & Eficacia del trabajo grupal & 0.008 & {$[-0.016 ; 0.033]$} \\
\hline \multirow{6}{*}{$\begin{array}{l}\text { Estrategia de } \\
\text { aprendizaje }\end{array}$} & Estrategias Motivacionales & $0.024^{*}$ & {$[0.006 ; 0.055]$} \\
\hline & Componentes afectivos & 0.008 & {$[-0.016 ; 0.033]$} \\
\hline & Estrategias metacognitivas & 0.021 & {$[-0.002 ; 0.05]$} \\
\hline & $\begin{array}{l}\text { Estrategias de control de contexto, interacción social y manejo de } \\
\text { recursos }\end{array}$ & 0.004 & {$[-0.006 ; 0.091]$} \\
\hline & Estrategia de búsqueda de selección & $0.049^{*}$ & {$[0.106 ; 0.089]$} \\
\hline & Estrategias relacionadas con el proceso de información & $0.060^{*}$ & {$[0.023 ; 0.098]$} \\
\hline \multirow{6}{*}{$\begin{array}{l}\text { Trabajo } \\
\text { Autónomo }\end{array}$} & Estrategias de Ampliación & 0.024 & {$[-0.006 ; 0.006]$} \\
\hline & Estrategia de Colaboración & 0.008 & {$[-0.016 ; 0.033]$} \\
\hline & Estrategia de Conceptualización & 0.211 & {$[-0.004 ; 0.046]$} \\
\hline & Estrategia de Planificación & 0.043 & {$[-0.006 ; 0.092]$} \\
\hline & Estrategia de Preparación de exámenes & $0.050^{*}$ & {$[0.011 ; 0.889]$} \\
\hline & Estrategia de Participación & $0.060^{*}$ & {$[0.232 ; 0.098]$} \\
\hline \multirow{3}{*}{$\begin{array}{l}\text { Competencia } \\
\text { Digital del } \\
\text { Estudiante }\end{array}$} & Medios Tecnológicos & $0.117^{*}$ & {$[0.031 ; 0.204]$} \\
\hline & Tiempo de uso del Internet & 0.005 & {$[-0.032 ; 0.043]$} \\
\hline & $\begin{array}{l}\text { Competencias en conocimiento y uso de las TIC en la comunicación } \\
\text { social y aprendizaje colaborativo }\end{array}$ & $0.122^{*}$ & {$[0.041 ; 0.254]$} \\
\hline \multirow{3}{*}{$\begin{array}{l}\text { Competencia } \\
\text { Digital del } \\
\text { Estudiante }\end{array}$} & $\begin{array}{l}\text { Competencia de uso de las TIC para la búsqueda y tratamiento de } \\
\text { información }\end{array}$ & $0.250^{*}$ & {$[0.095 ; 0.286]$} \\
\hline & $\begin{array}{l}\text { Competencias interpersonales en el uso de las TIC en el contexto } \\
\text { universitario }\end{array}$ & -0.005 & {$[-0.028 ; 0.016]$} \\
\hline & Herramientas virtuales y de comunicación social en Pregrado & $0.222^{*}$ & {$[0.151 ; 0.253]$} \\
\hline
\end{tabular}

Existen escasos estudios para contrastar con nuestros resultados; sin embargo, un estudio realizado en China sirvió de contraste, ya que mostró presentando una correlación significativa entre las estrategias de aprendizaje en procesos de información y el estilo activo aumentan el rendimiento académico de los estudiantes (Feng, et al., 2019). Estos resultados demuestran que las universidades que están ingresando con mayor cobertura a la educación virtual como se mencionó en el anterior modelo tienen la responsabilidad de generar programas e incentivar en los estudiantes este tipo de destrezas, especialmente en TIC; porque sin ello, sus posibilidades de poder adquirir información y realizar sus actividades propuestas dentro de un entorno virtual son casi nulas. 


\section{CONCLUSIONES}

De acuerdo con el estudio presentado y a los resultados obtenidos, se pueden plantear las siguientes conclusiones:

1) El modelo según variables macro mostró que las tres variables que más influyen en el rendimiento académico de los estudiantes del programa de educación, a partir de los factores educativos analizados en un entorno virtual son: estilo de aprendizaje, trabajo cooperativo y competencia digital incluyendo como moderadoras las variables docentes. 2) Las dimensiones que tienen mayor fuerza para lograr un buen rendimiento académico, teniendo como moderadoras las variables docentes son: conocimiento de las herramientas virtuales y de comunicación social de pregrado, y uso de medios tecnológicos, estilo de aprendizaje activo y reflexivo, estrategias de búsqueda de selección, concepción del trabajo en grupo, utilidades del trabajo en grupo para su formación, estrategias de participación.3) El mayor porcentaje de estudiantes de educación alcanzaron el nivel alto en las competencias genéricas discentes requeridas para el trabajo cooperativo, realizar las actividades propuestas con autonomía y el manejo de herramientas digitales; asimismo, lograron tener un buen rendimiento académico. 4) Los docentes evaluados del programa de educación continúan capacitándose para mejorar la preparación y desarrollo de las clases a través del entorno virtual, considerando las estrategias y estilos de aprendizaje de los estudiantes, de tal modo que alcancen el dominio de las competencias estudiadas. 5) Finalmente, este estudio primario realizado en el Perú podría servir para que se realicen futuras investigaciones, buscando generalizar y contrastar con los resultados presentados.

\section{REFERENCIAS}

Arbuthnott, K.D., y Krätzig, G.P., Effective teaching: sensory learning styles versus general memory processes, https://doi.org/10.2466/06.IT.4.2, Comprehensive Psychology, 4(2), 1-9 (2015)

Colonio, L.A., Estilos de aprendizaje y rendimiento académico de los estudiantes de los Cursos comprendidos dentro de la línea de construcción-DAC-FIC-UNI, http://repositorio.upch.edu.pe/handle/upch/3848, Universidad Peruana Cayetano Heredia, Lima, Perú (2017)

Chou, S.W., y Liu, C.H., Learning effectiveness in a web-based virtual learning environment: A learner control perspective, https://doi.org/10.1111/j.1365-2729.2005.00114.x, Journal of Computer Assisted Learning, 21(1), 65-76 (2005)

Dağ, F., y Geçer, A.K., Relations between online learning and learning styles, https://doi.org/10.1016/j.sbspro.2009.01.155, Procedia-Social and Behavioral Sciences, 21(1), 65-76 (2009)

Esteve-Mon, F.M., La competencia digital docente: Análisis de la autopercepción y evaluación del desempeño de los estudiantes universitarios de educación por medio de un entorno 3D, https://www.tdx.cat/handle/10803/291441\#page=1, Universidad Rovira i Virgili. Tarragona, España (2015)

Esteve-Mon, F.M., Angeles, M., y Adell-Segura, J., Digital competence and computational thinking of student teacher, https://doi.org/10.3991/ijet.v15i02.11588, International Journal of Emerging Technologies in Learning, 15(02), 29-41 (2020)

Feng, Y., Iriarte, F., y Cobos, J., Relationship between learning styles, learning strategies and academic performance of Chinese students who learn spanish as a foreign language, https://doi.org/10.1007/s40299-019-00496-8, The AsiaPacific Education Researcher, 29, 1-10 (2019)

García, M., González, I., y Mérida, R., Validación del cuestionario de evaluación ACOES. Análisis del trabajo cooperativo en educación superior, https://doi.org/10.6018/rie.30.1.114091, Revista de Investigación Educativa, 30 (1), 87-109 (2012)

García-Valcárcel, A., González, L., y otros 7 autores, Competencias en TIC y rendimiento académico en la universidad: diferencias por género, $1^{\circ}$ Ed.,1-312, Pearson Educación, Nacalpam de Juárez, México (2009)

Gargallo, B., Suárez-Rodríguez, J., y Pérez-Pérez, C., El cuestionario CEVEAPEU. Un Instrumento para la evaluación de las estrategias de aprendizaje de los estudiantes universitarios, https://doi.org/10.7203/relieve.15.2.4156, Revista Electrónica de Investigación y Evaluación Educativa, 15(2), 1-31 (2009)

Gargallo, B., Suárez, J., Garfella, P.R., y Fernández, A., El cuestionario CEMEDEPU. un Instrumento para la evaluación de la metodología docente y evaluativa de los profesores universitarios, Estudios sobre Educación, 21, 9-40 (2011)

Gómez-Galán, J., Media education in the ICT era: theorical structure for innovative theaching styles, https://doi.org/10.3390/info11050276, The Asia Pacific Education Researcher, 11(5), 276-293 (2020)

Gonzalez, T., De la Rubia M., y otros 5 autores, Influence of COVID-19 confinement on students' performance in higher education, https://doi.org/10.1371/journal.pone.0239490, PLoS One, 15(10), 1-23 (2020)

Henri, D.C., Morrell, L.J., y Scott, W.G., Student perceptions of their autonomy at University, https://doi.org/10.1007/s10734-017-0152-y, High Educ, 75, 507-516 (2018) 
Hughes, J.E., McLeod, S., y otros 3 autores, Academic achievement and perceptions of the learning environment in virtual and traditional secondary mathematics classrooms, http://dx.doi.org/10.1080/08923640701595365, American Journal of a Distance Education, 21(4),199-214 (2007)

Ilomäki, L., Paavola, S., Lakkala, M., y Kantosalo, A., Digital competence-an emergent boundary concept for policy and educational research, https://doi.org/10.1007/s10639-014-9346-4, Educ Inf Technol 21(3), 655-679 (2016)

Juárez, C., Propiedades psicométricas del cuestionario Honey-Alonso de estilos de aprendizaje (CHAEA) en una muestra mexicana, https://doi.org/10.7763/IJIET.2015.V5.501, Revista de Estilos de Aprendizaje, 7(13), 136-154 (2014)

Khan, M.J. y Rasheed, S., Moderating role of learning strategies between meta-cognitive awareness and study habits among university students, https://doi.org/10.33824/PJPR.2019.34.1.12, Pakistan Journal of Psychological Research, 34(1), 215-231 (2019)

López-Aguado, M., Diseño y análisis del cuestionario de estrategias de trabajo autónomo (CETA) para estudiantes universitarios, Revista de Psicodidáctica, 15(1), 77-99 (2010)

Mari, J.S., y Gumel, S.A., Effects of jigsaw model of cooperative learning on self-efficacy and achievement in chemistry among concrete and formal reasoners in colleges of education in Nigeria, https://doi.org/10.7763/IJIET.2015.V5.501, International Journal of Information and Education Technology, 5 (3), 196-199 (2015)

Martínez-Palmera, O., Combita-Niño, H., y De la Hoz-Franco, E., Mediación de los objetos virtuales de aprendizaje en el desarrollo de competencias matemáticas en estudiantes de ingeniería, https://dx.doi.org/10.4067/S071850062018000600063, Formación Universitaria, 11(6), 63-74 (2018)

$\mathrm{Ng}, \mathrm{K}$. y So, J., Impact of secondary education reform on tertiary students' generic competencies: a case study of associate degree freshmen in Hong Kong, https://doi.org/10.21153/jtlge2017vol8no1art633, Journal of Teaching and Learning for Graduate Employability, 8(1), 106-122 (2017)

Norzagaray, C., Sevillano, M., y Valenzuela, B., Estrategias de aprendizaje y rendimiento académico: La perspectiva del estudiante de psicología, http://dx.doi.org/10.4067/S0718-50062015000500007, Revista Lasallista de Investigación, 9(6), 47-56 (2013)

Pascual, M.A., Ortega-Carrillo, J.A., Pérez-Ferra, M., y Fombona, J., Competencias digitales en los estudiantes del grado de maestro de educación primaria. El caso de tres universidades españolas, https://dx.doi.org/10.4067/S071850062019000600141, Formación Universitaria, 12(6), 141-150 (2019)

Pettersson, F., On the issues of digital competence in educational contexts-a review of literature, https://doi.org/10.1007/s10639-017-9649-3, Educ Inf Technol, 23, 1005-1021 (2018)

Rahimi, S., Sohrabi, Y., Nafez, A., y Dabirian, M., Learning styles in university education (systematic review), https://doi.org/10.5958/0976-5506.2017.00146.2, Indian Journal of Public Health Research and Development. 8(2), 386 (2017)

Silva, J., Un modelo pedagógico virtual centrado en las E-actividades, https://doi.org/10.6018/red/53/10, Revista De Educación a Distancia, 17(53) (2017)

Tang, K., Personality traits, teamwork competencies and academic performance among first-year engineering students, https://doi.org/10.1108/HESWBL-11-2019-0153, Higher Education, Skills and Work-Based Learning, Vol. ahead-of-print No. ahead-of-print (2020)

Veytia, M., Propuesta para evaluar las competencias digitales en los estudiantes de postgrado que utilizan la plataforma moodle, XIV Encuentro Internacional Virtual Educa (2013)

Zempoalteca, B., Barragán, J.F., González, J., y Guzmán, T., Formación en TIC y competencia digital en la docencia en instituciones públicas de educación superior, http://dx.doi.org/10.32870/Ap.v9n1.922, Revista Apertura, 9(1), 80-96 (2017) 
